\title{
Implicit Cognition and Addiction: An Introduction
}

\author{
Reinout W. Wiers and Alan W. Stacy
}

\section{IMPLICIT COGNITION}

Until recently, most research on cognitive processes and drug abuse has focused on theories and methods of explicit cognition. When explicit cognition is assessed, people are asked directly to introspect about the causes of their behavior, usually through traditional questionnaires. It may be questioned, however, to what extent such methods reflect fundamental aspects of human cognition and motivation. Therefore, basic cognition researchers have turned to indirect methods to assess implicit cognitions, defined as "introspectively unidentified (or inaccurately identified) traces of past experience that mediate feeling, thought, or action" (Greenwald \& Banaji, 1995; see De Houwer, Chapter 2, for issues regarding the definition of implicit cognition). In this book, we use the term "implicit" to refer to indirect measures as well as to implicit, automatic processes that are likely assessed by these measures (cf. De Houwer, Chapter 2). Assessing implicit cognitions has several potential benefits:

1. Implicit measures may assess cognitive processes that are unavailable to introspection.
2. These approaches are less sensitive to self-justification and social desirability.

3. Implicit and explicit cognitions explain unique variance or different aspects of behavior.

4. Implicit cognition approaches provide a new important bridge between diverse disciplines as well as human and animal research on addiction.

In this handbook, research from a variety of relevant disciplines is brought together for the first time, including major cognitive and biological approaches to addiction, basic research on implicit cognition and dualprocess models, and implications of these new views for prevention and treatment are discussed. This is done by experts working in the addictions or in allied fields such as experimental psychopathology, health psychology, cognitive science, and neuroscience. As editors, we are very happy that many experts doing work in different areas of research (often not directly related to addiction) agreed to contribute to this book. The authors include an approximately equal representation of scholars from North 
America and Europe, where most of the research on implicit cognition and addiction has been conducted.

In many of the chapters, one of the systems that steers addictive behaviors is an associative system. The importance of associations or connections, broadly defined, can be traced from Aristotle, the British empiricists, and William James to contemporary work on connectionist and associative memory models of memory, modern learning theories, implicit social cognition, and neuroscience. A focus on connections among elements (e.g., concepts, affects, groups of neurons, etc.) is a different way of viewing the basis of cognition than is a focus on the elements themselves or stored facts/if-then rules about those elements (see Deutsch \& Strack and McEvoy $\&$ Nelson, Chapters 4 and 5, respectively). A simple way to think about connections is that a memory or cognition does not occur in isolation. It is usually triggered (activated or engaged) by something else, either in perception or memory. This "something else" must be connected to the memory or cognition to act as a trigger, and a trigger (e.g., seeing a bottle of wine) may have a hierarchy of strength of connections with other phenomena (e.g., negative affect, positive affect, arousal, nonaffective concepts, images, etc.). Indeed, there is also evidence that associations may automatically trigger actions in the absence of conscious recollection or intentional retrieval (see Palfai, Chapter 26). Automatic activation could occur through a number of different architectures, which have different ways of modeling connections and the operation of activation (e.g., Hintzman, 1990; Smith \& DeCoster, 1998). Some of these architectures can readily model higherorder cognitions such as schemas (Hintzman, 1986) or emergent properties of cognition (Bechtel \& Abrahamsen, 2002), revealing that patterns of interconnection and activation across multiple units constitute more than "simple associationism."
Associations, whether measured with reaction time (RT) tests, word association, attentional bias, or other tests outlined in this book or inferred from biological measures have different implications than the storage of facts or rules. Connections involving implicit systems or processes may often have the following characteristics: They take time to establish or strengthen; once established, they operate autonomously when engaged, not requiring the intervention of other systems; they are relatively resistant to change (although they are sensitive to context effects; see Deutsch \& Strack, Chapter 4; Krank \& Wall, Chapter 19); and they originate from both cultural and personal experiences, including experiences involving reinforcement and affect (see McEvoy \& Nelson, Chapter 5; Stacy et al., Chapter 6).

Finally, many authors have come to the conclusion that there must be a second system, or set of systems, that is rule-based and has limited capacity and that this second system also has some influence on behavior (e.g., Bechara et al., Chapter 15; Deutsch \& Strack, Chapter 4; Evans \& Coventry, Chapter 3; Wiers et al., Chapter 22; Yin \& Knowlton, Chapter 12). An important issue, also for interventions in this domain, concerns the interplay between these systems. From this perspective, there are different ways to change addictive behaviors. First, one may attempt to change something in the automatic system. Modern learning psychology has indicated that extinction of once-established associations may be difficult if not impossible (see Hermans \& Van Gucht, Chapter 32; Wiers et al., 2004). There may, however, be viable alternatives, such as increasing aversive associations (which has an old tradition; see Wiers et al., Chapter 22), strengthening associations between triggers of addictive behaviors with alternative behaviors (e.g., Palfai, Chapter 26; Prestwich et al., Chapter 29), or "attentional retraining" procedures (see de Jong et al., Chapter 27; Fadardi et al., Chapter 9). 
Second, there may be ways to use the second rule-based system in changing behavior. Mere inhibition of impulses from the associative system may be counterproductive (see Palfai, Chapter 26), but this system may be used to establish alternative associations (e.g., Palfai, Chapter 26) or to counter the influence of the associations on behavior (e.g., Marlatt \& Ostafin, Chapter 33).

\section{ADDICTION}

Before turning to a brief introduction of the sections in this book, the second part of the title of the book may need some clarification. Why did we use the term "addiction" and not "substance dependence," as current psychiatric classification schemas would want us to categorize the disorders central in this book? The first reason is that we did not want to restrict ourselves to addictive behaviors that involve substances, because interesting work is done with respect to other addictions, such as gambling (see Evans \& Coventry, Chapter 3; Zack \& Poulos, Chapter 24). Second, some of the applications in the book refer to prevention of addictions; also from that perspective the general term addiction seemed most appropriate. Third, in many current conceptualizations of addiction (unlike current psychiatric classification schemes) appetitive motivations are important (Robinson \& Berridge, 2003; Stewart et al., 1984; Wise and Bozarth, 1987). The related concept of "incentive sensitization" (Robinson \& Berridge, 1993, 2003), based on work in animal models, has stirred enthusiasm in recent theorizing about implicit cognitive processes involved in addiction. Sensitization refers to the hypersensitization of mesolimbic circuits to drug effects and drug-associated stimuli. Robinson and Berridge (1993, 2003) proposed that psychologically this leads to the excessive attribution of incentive salience to drug-related representations, causing pathological "wanting.” Implicit "wanting” is "similar to implicit memory and to unconscious perception (e.g., blindsight), which can occur and influence behavior without conscious awareness" (Robinson \& Berridge, 2003, p. 36). Implicit "wanting" need not lead to subjective wanting, and can influence behavior without it (see also Berridge \& Robinson, Chapter 31). The incentive-sensitization theory has been linked to a variety of implicit cognitive mechanisms in addiction, including the most dominant ones: attentional bias and implicit drug associations (e.g., Chapters 10, 11, 17, 21, 22, 24, and 27). In addition, important transfer effects have been noted in animals (e.g., crosssensitization, see Chapter 31) and recently similar findings have been reported in humans between different drugs (Ostafin $\&$ Palfai, Chapter 25) and between drugs and gambling (Zack \& Poulos, Chapter 24), underscoring the use of "addiction" rather than substance dependence (note that the concepts of incentive motivation and sensitization are not represented in current psychiatric classification schemes). The link between neurobiological work on sensitization and implicit cognition in human addictions emphasizes the potential bridging function of implicit cognition in addiction, where so far human and animal work have been relatively isolated.

\section{GENERAL OUTLINE OF THE BOOK}

The book consists of seven sections.

Section I focuses on general theoretical issues regarding implicit and explicit cognition in general and in relation to addiction. In Chapter 2, De Houwer argues that implicit measures are measurement outcomes that have certain functional properties that should be critically evaluated, as has been done for the related concept of automaticity. He concludes that to the extent that implicit measures 
reflect the automatic impact of attitudes and cognitions, they could provide a unique insight into the effects of automatic processing on real-life behavior. As noted above, many researchers have turned to the use of implicit measures from a dual-process perspective. The general idea is that human behavior is steered by two relatively independent systems, one of which can be characterized as fast, automatic, and associative, whereas the other is relatively slow, effortful, and controlled (Deutsch \& Strack, Chapter 4; Evans \& Coventry, Chapter 3; Strack \& Deutsch, 2004). Emphasizing the relative independence of the processes, Evans (2003) paraphrased them as "two minds in one head." From this perspective, the use of implicit measures may provide a unique window to tap into the fast, automatic, and associative processes that partly steer behavior (especially under circumstances when effortful control processes are difficult to engage; cf. Fillmore \& Vogel-Sprott, Chapter 20). The chapters by Evans and Coventry (Chapter 3) and Deutsch and Strack (Chapter 4) have been inspired by research in the areas of cognitive and social psychology. Both chapters present general functional dualprocess models and apply them to addiction. Both models are functional in the sense that they do not directly link the proposed systems to underlying neurobiological mechanisms (cf. Chapters 12 and 15 in Section III for neurobiologically based multiple-process models). Another area of research in which the implicitexplicit distinction has been prominent is memory research. McEvoy and Nelson (Chapter 5) review different techniques for studying implicit memory: the methods of savings and of indirect test instructions, and the process dissociation procedure (see also Chapter 20). They then describe their model of cued recall that incorporates both implicit and explicit memory processes (PIER2, which is described in Chapter 5; Nelson et al., 1998), and illustrate it with examples relevant to addictive behaviors.
Section II focuses on assessment paradigms and their theoretical basis. In the first chapter in this section, Stacy et al. (Chapter 6) discuss basic memory research on word association and illustrate how several well-supported cognitive models (such as PIER2) can use these assessments of association to help explain and understand addictive behaviors. In Chapter 7, Houben et al. describe a different assessment approach: reaction time tests of associations that have generated much recent enthusiasm about implicit measures. They describe several of the major reaction time tests, including different recent versions of the Implicit Association Test (IAT), the Extrinsic Affective Simon Task (EAST), and several semantic and affective priming tests, with applications to addiction. In Chapter 8, Goldman et al. group many different assessment paradigms under the umbrella of their general expectancy theory, contending that expectancies can be assessed with either direct or indirect methods. In this approach, "expectancies" are seen as reflections of different processing systems that are shaped by evolution to anticipate the future. In Chapter 9, Fadardi et al. discuss individualized versus general measures of implicit cognition. This issue is relevant for whatever assessment tool is used, because the assessment may show utility only when the stimuli are tailored to the individual. The final two chapters of Section II both focus on measures of attentional bias. Bruce and Jones (Chapter 10) review methods used to assess and understand attentional bias in addiction, with a focus on the use of the emotional or drugrelated Stroop paradigm. The emerging picture over different tests is that there might be a continuity of attentional bias along the consumption continuum from light to heavy use to problematic use (but see Chapter 21). Field et al. (Chapter 11) review models that assume that attentional biases occur at early stages of stimulus processing, and that attentional biases are associated with subjective 
craving and the tendency to approach drug-related cues and discuss relevant empirical findings. These include studies that have used visual-probe tasks and eye movement monitoring techniques to investigate the component processes of biases in visual orienting to drug-related stimuli (attracting vs. maintaining attention). It is argued that attentional biases in addictive and appetitive behaviors are qualitatively different from those in anxiety. Despite their differences, each of the strategies outlined in these chapters converge on assessing fundamental cognitive and attentional biases and these biases are likely to channel behavior down certain routes, in either harmful or beneficial directions.

Section III focuses on brain mechanisms underlying addiction. In Chapter 12, Yin and Knowlton outline different types of learning and their different neural substrates (circuits rather than structures) and the different roles these systems play in addictive behaviors. In Chapter 13, Franken et al. review findings in humans with recent functional imaging techniques, concerning the neural correlates (neuroanatomy and neurotransmitters) of general psychological processes that play a role in addiction: reward, craving, attention, memory, and decision making. In Chapter 14, Mucha et al. discuss other physiological measures that can be used in humans to assess drug-related motivation in an indirect way, with an emphasis on the startle-response measures. A common theme is that indices of brain functioning in addiction in humans (e.g., psychophysiological measures, functional imaging techniques) can be viewed as another indirect way to assess motivation underlying addiction. There has been, however, a paucity of studies directly comparing indirect brain measures and indirect cognitive measures. In Chapter 15, Bechara et al. propose their neurologically inspired dualprocess model to explain addictive behaviors. They present results that indicate that at least three different (nonexclusive) processes can be involved in addictions: strong automatic appetitive associations, two different problems in the reflective system, and problems related to decision making and to impulse control. These different neurological problems may characterize subgroups of individuals prone to addictions. In the last chapter of Section III, Curtin et al. (Chapter 16) present a model of implicit and explicit motivational processes in drug use, integrating basic neuroscience research on cognitive control and research on bottom-up motivational processes. It is argued that both positive (approach) and negative reinforcement (withdrawal) motivation can be triggered automatically, and that craving is a function of cognitive control systems reacting to automatically activated tendencies to use drugs (cf. Tiffany, 1990). The model further states that other situations that recruit cognitive control will result in craving, such as response conflict and novel or unfavorable outcomes. This chapter provides a bridge to the next section.

Section IV presents work on the interplay between implicit and explicit cognitive processes, emotion and motivation, and context effects in addictive behaviors. In Chapter 17, Cox et al. present their motivational theory of current concerns. Current concerns influence behavior by keeping an individual oriented toward cues and actions that advance the attainment of the goal. The underlying process is thought to be unconscious, but goals usually become conscious. Applied to addictive behaviors, performing addictive behaviors to regulate one's emotions can become an automatized goal, resulting in an attentional bias. This model has important implications for treatment: It is important to set alternative goals (e.g., through motivational techniques) and the attentional bias may be unlearned (e.g., through attentional retraining). Regarding the association between emotions and drug use, Birch et al. (Chapter 18) conclude that there is little evidence for 
simple relationships. Models that take into account moderators like individual differences in drug-use motives, however, better explain the data. Motivational theories generally distinguish between two broad classes of motivation for drug use: positive and negative reinforcement (e.g., Cooper et al., 1995; Curtin et al., Chapter 16), and the scarce evidence is consistent with the idea that in individuals who have strong enhancement motives, positive emotions activate both implicit and explicit motivations to use alcohol or drugs. For negative reinforcement (drinking to relieve tension or negative affect), however, results are less consistent: A negative mood has been found to activate explicit but not implicit coping motives (Birch et al., Chapter 18; cf. Wiers et al., Chapter 22). Context has been known for some time to affect cognition, memory, and drug-use behaviors. Krank and Wall (Chapter 19) review and integrate this literature. Although "context" is usually thought of in physical terms, an emotion can also function as a context for drug use, and recent evidence suggests that context also plays an important role in assessment, both for more implicit (e.g., Mitchell et al., 2003) and more explicit assessment methods (e.g., Schwartz, 1999). In Chapter 20, Fillmore and VogelSprott review the effects of alcohol and other drugs on the relative contributions of automatic versus controlled processes using the process dissociation approach. It is argued that the ability of drugs to promote a reliance on automatic influences could explain a broad range of behavioral effects observed in the drugged state, and provide new insights into factors that contribute to drug abuse.

Section $\mathrm{V}$ is organized by addictive behavior. In Chapter 21, Waters and Sayette systematically review the literature on automatic affective and motivational processes in smoking. They conclude that there are robust differences regarding an attentional bias for smoking-related stimuli in smokers, not found in nonsmokers, but that there appears to be no strong relation with heaviness of smoking or abstinence (cf. Chapter 10). In Chapter 22, Wiers et al. review the work on implicit cognition in relation to alcohol use and abuse, using the three broad categories of alcohol-related cognitions that have emerged from research using explicit measures: positive and negative reinforcement and negative expectancies. Most research has focused on positive reinforcement, revealing ample evidence for automatic appetitive reactions in heavy and problem drinkers (attentional bias, automatic arousal or approach associations). In addition, there is more scattered evidence for the existence of automatic negative associations. It is argued that implicit negative reinforcement expectancies may be difficult to assess because in contrast to positive and negative associations, they involve two different associations. Ames et al. (Chapter 23) review literature on implicit cognitions in drugs of abuse with an emphasis on substances other than alcohol and cigarettes. For a variety of substances, implicit memory association measures and reaction time measures have been found to successfully predict addictive behaviors and to predict a different portion of the variance in behavior than explicit measures. Ames and her colleagues also point at the importance of implementing these measures in intervention research. As indicated earlier, the other two chapters of this section review recent work on implicit cognition and gambling (Zack \& Poulos, Chapter 24) and cross-drug effects (Ostafin \& Palfai, Chapter 25).

Section VI focuses on implications of the implicit cognition approach for interventions (prevention and treatment) in addiction (readers interested in this topic should note that many chapters outside this section also make useful suggestions for interventions). In the first chapter of this section, Palfai (Chapter 26) discusses self-regulation in addictive 
behaviors. Usually self-regulation is seen as the product of conscious, controlled processing, which serves to counter impulsive tendencies to use drugs (cf. Chapters 3, 4, 15). Recent research in social cognition indicates, however, that automatic processes play an important role in self-regulation (in line with the automatic pursuit of goals proposed in Chapter 17). From this perspective, new strategies for interventions can be derived to harness implicit processes in support of alternative goals and behaviors. De Jong et al. (Chapter 27) review work in other areas of psychopathology (anxiety and eating disorders) on the role of implicit cognition in the etiology and maintenance of problem behaviors. Given promising results in these other areas, they suggest the possibility of attentional retraining in addiction interventions, a topic currently investigated in several labs. In Chapter 28, Krank and Goldstein focus on how implicit cognition can be used in understanding the etiology and prevention of addictive behaviors in adolescence. Importantly, they indicate that implicit cognition measures can be used to prospectively predict changes in drug-use behavior (alcohol and marijuana), including early transitional stages. They also review suggestions on countering the influence of implicit drug associations and the influence of alcohol advertising (e.g., inoculation training). In the final chapter of this section, Prestwich et al. (Chapter 29) review the recent literature on implementation intentions, an example of a specific form of planning (in ifthen format) that has been found to successfully influence a variety of health behaviors in an automatic way. The application of this and other strategies in this section to addictive behavior provides an exciting new opportunity to improve intervention effects.

Section VII contains invited commentaries by outside experts not working directly on implicit cognition and addiction (except for one coauthor, Ostafin). Commentaries are provided by Sher, a leader in research on the origins of addictive behaviors in humans (Chapter 30); by Berridge and Robinson, two of the leading researchers on neurobiological processes in addiction (Chapter 31); by Hermans and Van Gucht, experts on learning theory and psychopathology (Chapter 32); and by Marlatt (with Ostafin, Chapter 33), a leader in relapse prevention, who discusses clinical applications of implicit cognition. In the final chapter (34) we give our concluding comments, which address some common themes, directions for the future, and challenges.

\section{FINAL NOTE}

We hope that this volume will stimulate further research on implicit cognition and addiction. All chapters have been reviewed by the editors and at least two other peer reviewers, usually authors from other (related) chapters and in some cases outside experts. We believe this procedure has increased the quality of the chapters and stimulated their integration. As the contents of this volume illustrate, implicit cognition is a rapidly growing direction in addiction research. This is not surprising, given the current interest in implicit cognition in general and the fact that addictive behaviors strike many as "irrational" behaviors par excellence. We hope this book will help to further integrate research from different independent fields that are all relevant for a better understanding of the etiology, maintenance, and prevention or treatment of addictive behaviors. We hope that this book will both stimulate further research and theorizing in this area and provide the groundwork for new approaches in the prevention and treatment of addictive behaviors. Finally, we thank all authors for their contributions and reviews, and acknowledge the outstanding editorial assistance of James Pike, Brian Houska, and Deborah Jelinek. We also acknowledge support from grants from the National Science Foundation of The 
Netherlands (N.W.O.) VIDI grant 452.03.005 and from the National Institute on Drug Abuse
(DA 16094) for research by the editors and by several of the chapter authors.

\section{REFERENCES}

Bechtel, W., \& Abrahamsen, A. (2002). Connectionism and the mind: Parallel processing, dynamics, and evolution in networks (2nd ed.). Malden, MA: Blackwell.

Cooper, M. L., Frone, M. R., Russell, M., \& Mudar, P. (1995). Drinking to regulate positive and negative emotions: A motivational model of alcohol use. Journal of Personality and Social Psychology, 69, 990-1005.

Evans, J. St. B. T. (2003). In two minds: Dual-process accounts of reasoning. Trends in Cognitive Science, 7(10), 454-459.

Greenwald, A. G., \& Banaji, M. R. (1995). Implicit social cognition: Attitudes, self-esteem, and stereotypes. Psychological Review, 102(1), 4-27.

Hintzman, D. L. (1986). "Schema abstraction" in a multiple-trace memory model. Psychological Review, 93(4), 411-428.

Hintzman, D. L. (1990). Human learning and memory: Connections and dissociations. Annual Review of Psychology, 41, 109-139.

Mitchell, J. P., Nosek, B. A., \& Banaji, M. R. (2003). Contextual variations in implicit evaluation. Journal of Experimental Psychology, 132, 455-469.

Nelson, D. L., McKinney, V. M., Gee, N. R., \& Janczura, G. A. (1998). Interpreting the influence of implicitly activated memories on recall and recognition. Psychological Review, 105, 299-324.

Robinson, T. E., \& Berridge, K. C. (1993). The neural basis of drug craving: An incentivesensitization theory of addiction. Brain Research Reviews, 18, 247-291.

Robinson, T. E., \& Berridge, K. C. (2003). Addiction. Annual Review of Psychology, $54,25-53$.

Schwartz, N. (1999). Self-reports. How questions shape the answers. American Psychologist, 54, 93-105.

Smith, E. R., \& DeCoster, J. (1998). Knowledge acquisition, accessibility, and use in person perception and stereotyping: Simulation with a recurrent connectionist network. Journal of Personality and Social Psychology, 74(1), 21-35.

Stewart, J., de Wit, H., \& Eikelboom, R. (1984). The role of unconditioned and conditioned drug effects in the self-administration of opiates and stimulants. Psychological Review, 91, 251-268.

Strack, F., \& Deutsch, R. (2004). Reflective and impulsive determinants of social behavior. Personality and Social Psychology Review, 3, 220-247.

Tiffany, S.T. (1990). A cognitive model of drug urges and drug-use behavior: Role of automatic and nonautomatic processes. Psychological Review, 97, 147-168.

Wiers, R. W., de Jong, P. J., Havermans, R., \& Jelicic, M. (2004). How to change implicit drug-related cognitions in prevention: A transdisciplinary integration of findings from experimental psychopathology, social cognition, memory and learning psychology. Substance Use \& Misuse, 39, 1625-1684.

Wise, R., \& Bozarth, M. (1987). A psychomotor stimulant theory of addiction. Psychological Review, 94, 469-492. 